\title{
Education in Twins and Their Parents Across Birth Cohorts Over 100 years: An Individual-Level Pooled Analysis of 42-Twin Cohorts
}

Karri Silventoinen, ${ }^{1,2}$ Aline Jelenkovic, ${ }^{1,3}$ Antti Latvala, ${ }^{4,5}$ Reijo Sund, ${ }^{1,6}$ Yoshie Yokoyama, $^{7}$ Vilhelmina Ullemar, ${ }^{8}$ Catarina Almqvist, ${ }^{8,9}$ Catherine A. Derom, ${ }^{10,11}$ Robert F. Vlietinck, ${ }^{10}$ Ruth J. F. Loos, ${ }^{12}$ Christian Kandler, ${ }^{13}$ Chika Honda, ${ }^{2}$ Fujio Inui, ${ }^{2,14}$ Yoshinori Iwatani, ${ }^{2}$ Mikio Watanabe, ${ }^{2}$ Esther Rebato, ${ }^{3}$ Maria A. Stazi, ${ }^{15}$ Corrado Fagnani, ${ }^{15}$ Sonia Brescianini, ${ }^{15}$ Yoon-Mi Hur, ${ }^{16}$ Hoe-Uk Jeong, ${ }^{16}$ Tessa L. Cutler, ${ }^{17}$ John L. Hopper, ${ }^{17,18}$ Andreas Busjahn, ${ }^{19}$ Kimberly J. Saudino, ${ }^{20}$ Fuling Ji, ${ }^{21}$ Feng Ning, ${ }^{21}$ Zengchang Pang, ${ }^{21}$ Richard J. Rose ${ }^{22}$ Markku Koskenvuo, ${ }^{4,5}$ Kauko Heikkilä, ${ }^{4,5}$ Wendy Cozen, ${ }^{23,24}$ Amie E. Hwang, ${ }^{23}$ Thomas M. Mack, ${ }^{23,24}$ Sisira H. Siribaddana, ${ }^{25,26}$

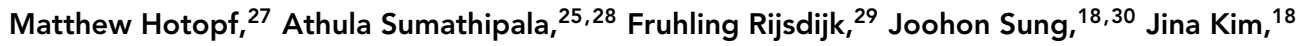
Jooyeon Lee, ${ }^{18}$ Sooji Lee, ${ }^{18}$ Tracy L. Nelson, ${ }^{31}$ Keith E. Whitfield, ${ }^{32}$ Qihua Tan, ${ }^{33}$ Dongfeng Zhang, ${ }^{34}$ Clare H. Llewellyn, ${ }^{35}$ Abigail Fisher, ${ }^{35}$ S. Alexandra Burt, ${ }^{36}$ Kelly L. Klump, ${ }^{36}$ Ariel Knafo-Noam, ${ }^{37}$ David Mankuta, ${ }^{38}$ Lior Abramson, ${ }^{37}$ Sarah E. Medland, ${ }^{39}$ Nicholas G. Martin, ${ }^{39}$ Grant W. Montgomery, ${ }^{40}$ Patrik K. E. Magnusson, ${ }^{8}$ Nancy L. Pedersen, ${ }^{8}$ Anna K. Dahl Aslan, ${ }^{8,41}$ Robin P. Corley, ${ }^{42}$ Brooke M. Huibregtse, ${ }^{42}$ Sevgi Y. Öncel, ${ }^{43}$ Fazil Aliev, ${ }^{44,45}$ Robert F. Krueger, ${ }^{46}$ Matt McGue, ${ }^{46}$ Shandell Pahlen, ${ }^{46}$ Gonneke Willemsen, ${ }^{47}$ Meike Bartels, ${ }^{47}$ Catharina E. M. van Beijsterveldt,${ }^{47}$ Judy L. Silberg, ${ }^{48}$ Lindon J. Eaves, ${ }^{48}$ Hermine H. Maes, ${ }^{49}$ Jennifer R. Harris, ${ }^{50}$ Ingunn Brandt, ${ }^{50}$ Thomas S. Nilsen, ${ }^{50}$ Finn Rasmussen, ${ }^{51}$ Per Tynelius, ${ }^{52}$ Laura A. Baker, ${ }^{53}$ Catherine Tuvblad, ${ }^{53,54}$ Juan R. Ordoñana, 55,56 Juan F. Sánchez-Romera, ${ }^{55,56}$ Lucia Colodro-Conde, ${ }^{55,57}$ Margaret Gatz, ${ }^{8,53}$ David A. Butler, ${ }^{58}$ Paul Lichtenstein, ${ }^{8}$ Jack H. Goldberg, ${ }^{59}$ K. Paige Harden, ${ }^{60}$ Elliot M. Tucker-Drob, ${ }^{60}$ Glen E. Duncan, ${ }^{61}$ Dedra Buchwald, ${ }^{61}$ Adam D. Tarnoki, ${ }^{62,63}$ David L. Tarnoki, ${ }^{62,63}$ Carol E. Franz, ${ }^{64}$ William S. Kremen, ${ }^{64,65}$ Michael J. Lyons, ${ }^{66}$ José A. Maia, $^{67}$ Duarte L. Freitas, ${ }^{68}$ Eric Turkheimer, ${ }^{69}$ Thorkild I. A. Sørensen, ${ }^{70}$ Dorret I. Boomsma, ${ }^{47}$ and Jaakko Kaprio ${ }^{4,5}$

${ }^{1}$ Department of Social Research, University of Helsinki, Helsinki, Finland

${ }^{2}$ Osaka University Graduate School of Medicine, Osaka University, Osaka, Japan

${ }^{3}$ Department of Genetics, Physical Anthropology and Animal Physiology, University of the Basque Country UPV/EHU, Leioa, Spain

${ }^{4}$ Institute for Molecular Medicine FIMM, Helsinki, Finland

${ }^{5}$ Department of Public Health, University of Helsinki, Helsinki, Finland

${ }^{6}$ Institute of Clinical Medicine, University of Eastern Finland, Kuopio, Finland

${ }^{7}$ Department of Public Health Nursing, Osaka City University, Osaka, Japan

${ }^{8}$ Department of Medical Epidemiology and Biostatistics, Karolinska Institutet, Stockholm, Sweden

${ }^{9}$ Pediatric Allergy and Pulmonology Unit at Astrid Lindgren Children's Hospital, Karolinska University Hospital, Stockholm, Sweden

${ }^{10}$ Centre of Human Genetics, University Hospitals Leuven, Leuven, Belgium

${ }^{11}$ Department of Obstetrics and Gynaecology, Ghent University Hospitals, Ghent, Belgium

${ }^{12}$ The Charles Bronfman Institute for Personalized Medicine, The Mindich Child Health and Development Institute, Icahn School of Medicine at Mount Sinai, New York, NY, USA

${ }^{13}$ Department of Psychology, MSB Medical School Berlin, School of Health and Medicine, Berlin, Germany 
${ }^{14}$ Faculty of Health Science, Kio University, Nara, Japan

${ }^{15}$ Istituto Superiore di Sanità - Centre for Behavioural Sciences and Mental Health, Rome, Italy

${ }^{16}$ Department of Education, Mokpo National University, Jeonnam, South Korea

${ }^{17}$ The Australian Twin Registry, Centre for Epidemiology and Biostatistics, The University of Melbourne, Melbourne, Australia

${ }^{18}$ Department of Epidemiology, School of Public Health, Seoul National University, Seoul, South Korea

${ }^{19}$ HealthTwiSt GmbH, Berlin, Germany

${ }^{20}$ Department of Psychological and Brain Sciencies, Boston University, Boston, MA, USA

${ }^{21}$ Department of Noncommunicable Diseases Prevention, Qingdao Centers for Disease Control and Prevention, Qingdao, China

${ }^{22}$ Department of Psychological and Brain Sciences, Indiana University, Bloomington, IN, USA

${ }^{23}$ Department of Preventive Medicine, Keck School of Medicine of USC, University of Southern California, Los Angeles,

CA, USA

${ }^{24}$ USC Norris Comprehensive Cancer Center, Los Angeles, CA, USA

${ }^{25}$ Institute of Research \& Development, Battaramulla, Sri Lanka

${ }^{26}$ Faculty of Medicine \& Allied Sciences, Rajarata University of Sri Lanka Saliyapura, Sri Lanka

${ }^{27}$ NIHR Mental Health Biomedical Research Centre, South London and Maudsley NHS Foundation Trust, and Institute of Psychiatry, Psychology and Neuroscience, King's College London, London, UK

${ }^{28}$ Research Institute for Primary Care and Health Sciences, School for Primary Care Research (SPCR), Faculty of Health, Keele University, Staffordshire, UK

${ }^{29}$ MRC Social, Genetic \& Developmental Psychiatry Centre, Institute of Psychiatry, Psychology and Neuroscience, King's College London, London, UK

${ }^{30}$ Institute of Health and Environment, Seoul National University, Seoul, South Korea

${ }^{31}$ Department of Health and Exercise Sciences and Colorado School of Public Health, Colorado State University, Fort

Collins, CO, USA

${ }^{32}$ Psychology and Neuroscience, Duke University, Durham, NC, USA

${ }^{33}$ Department of Public Health, Epidemiology, Biostatistics and Biodemography, University of Southern Denmark,

Odense, Denmark

${ }^{34}$ Department of Public Health, Qingdao University Medical College, Qingdao, China

${ }^{35}$ Health Behaviour Research Centre, Department of Epidemiology and Public Health, Institute of Epidemiology and

Health Care, University College London, London, UK

${ }^{36}$ Michigan State University, East Lansing, MI, USA

${ }^{37}$ The Hebrew University of Jerusalem, Jerusalem, Israel

${ }^{38}$ Hadassah Hospital Obstetrics and Gynecology Department, Hebrew University Medical School, Jerusalem, Israel

${ }^{39}$ Genetic Epidemiology Department, OIMR Berghofer Medical Research Institute, Brisbane, Australia

${ }^{40}$ Molecular Epidemiology Department, QIMR Berghofer Medical Research Institute, Brisbane, Australia

${ }^{41}$ Institute of Gerontology and Aging Research Network - Jönköping (ARN-J), School of Health and Welfare Jönköping

University, Jönköping, Sweden

${ }^{42}$ Institute for Behavioral Genetics, University of Colorado, Boulder, CO, USA

${ }^{43}$ Department of Statistics, Faculty of Arts and Sciences, Kırıkkale University, Kırıkkale, Turkey

${ }^{44}$ Psychology and African American Studies, Virginia Commonwealth University, Richmond, VA, USA

${ }^{45}$ Faculty of Business, Karabuk University, Turkey

${ }^{46}$ Department of Psychology, University of Minnesota, Minneapolis, MN, USA

${ }^{47}$ Department of Biological Psychology, VU University Amsterdam, Amsterdam, the Netherlands

${ }^{48}$ Department of Human and Molecular Genetics, Virginia Institute for Psychiatric and Behavioral Genetics, Virginia Commonwealth University, Richmond, VA, USA

${ }^{49}$ Department of Human and Molecular Genetics, Psychiatry and Massey Cancer Center, Virginia Commonwealth

University, Richmond, VA, USA

${ }^{50}$ Norwegian Institute of Public Health, Oslo, Norway

${ }^{51}$ Lund University, Lund, Sweden

${ }^{52}$ Department of Public Health Sciences, Karolinska Institutet, Stockholm, Sweden

${ }^{53}$ Department of Psychology, University of Southern California, Los Angeles, CA, USA

${ }^{54}$ School of Law, Psychology and Social Work/Criminology, Örebro University, Örebro, Sweden

${ }^{55}$ Department of Human Anatomy and Psychobiology, University of Murcia, Murcia, Spain

${ }^{56}$ IMIB-Arrixaca, Murcia, Spain

${ }^{57}$ QIMR Berghofer Medical Research Institute, Brisbane, Australia

${ }^{58} \mathrm{Health}$ and Medicine Division, The National Academies of Sciences, Engineering, and Medicine, Washington, DC, USA

${ }^{59}$ Department of Epidemiology, School of Public Health, University of Washington, Seattle, WA, USA

${ }^{60}$ Department of Psychology, University of Texas at Austin, Austin, TX, USA

${ }^{61}$ Washington State Twin Registry, Washington State University - Health Sciences Spokane, Spokane, WA, USA

${ }^{62}$ Department of Radiology, Semmelweis University, Budapest, Hungary

${ }^{63}$ Hungarian Twin Registry, Budapest, Hungary

${ }^{64}$ Department of Psychiatry, University of California, San Diego, CA, USA

${ }^{65}$ VA San Diego Center of Excellence for Stress and Mental Health, La Jolla, CA, USA

${ }^{66}$ Department of Psychology, Boston University, Boston, MA, USA

${ }^{67}$ CIFI2D, Faculty of Sport, University of Porto, Porto, Portugal

${ }^{68}$ Department of Physical Education and Sport, University of Madeira, Funchal, Portugal

${ }^{69}$ Department of Psychology, University of Virginia, Charlottesville, VA, USA

${ }^{70}$ Novo Nordisk Foundation Centre for Basic Metabolic Research (Section on Metabolic Genetics), and Department of

Public Health, Faculty of Health and Medical Sciences, University of Copenhagen, Copenhagen, Denmark 


\begin{abstract}
Whether monozygotic (MZ) and dizygotic (DZ) twins differ from each other in a variety of phenotypes is important for genetic twin modeling and for inferences made from twin studies in general. We analyzed whether there were differences in individual, maternal and paternal education between $\mathrm{MZ}$ and $\mathrm{DZ}$ twins in a large pooled dataset. Information was gathered on individual education for 218,362 adult twins from 27 twin cohorts (53\% females; $39 \% \mathrm{MZ}$ twins), and on maternal and paternal education for 147,315 and 143,056 twins respectively, from 28 twin cohorts (52\% females; $38 \% \mathrm{MZ}$ twins). Together, we had information on individual or parental education from 42 twin cohorts representing 19 countries. The original education classifications were transformed to education years and analyzed using linear regression models. Overall, $\mathrm{MZ}$ males had $0.26(95 \% \mathrm{Cl}[0.21,0.31])$ years and $\mathrm{MZ}$ females $0.17(95 \% \mathrm{Cl}[0.12,0.21])$ years longer education than DZ twins. The zygosity difference became smaller in more recent birth cohorts for both males and females. Parental education was somewhat longer for fathers of DZ twins in cohorts born in $1990-1999(0.16$ years, $95 \% \mathrm{Cl}[0.08,0.25])$ and 2000 or later $(0.11$ years, $95 \% \mathrm{Cl}[0.00,0.22])$, compared with fathers of $M Z$ twins. The results show that the years of both individual and parental education are largely similar in $\mathrm{MZ}$ and $\mathrm{DZ}$ twins. We suggest that the socio-economic differences between $\mathrm{MZ}$ and $\mathrm{DZ}$ twins are so small that inferences based upon genetic modeling of twin data are not affected.
\end{abstract}

Keywords: twins, zygosity, education, parental education

Understanding how monozygotic (MZ) and dizygotic (DZ) twins differ from each other has important methodological and possible public health implications. Quantitative genetic twin models assume that MZ and DZ twins are representative of the same background population (Posthuma et al., 2003). If they are not, this may be seen as differences in the means and variances between the two zygosity groups. Zygosity differences in anthropometric measures, especially in early life, are well documented: MZ twins weigh less and are shorter at birth than DZ twins (Hur et al., 2005). Furthermore, DZ twins were slightly taller and had a somewhat higher body mass index (BMI) than MZ twins in a large international twin study based on the same database also used in the present study. The differences were largest in childhood and decreased in adulthood, where differences were less than $1 \mathrm{~cm}$ in height and $0.1 \mathrm{~kg} / \mathrm{m}^{2}$ in BMI (Jelenkovic et al., 2015). A Swedish study of young adult men also found that MZ twins had slightly less muscle strength than DZ twins (Silventoinen et al., 2008).

Socio-economic status (SES) is an important determinant of health (Mackenbach et al., 2008), and education is one of the most important dimensions of SES in modern societies (Hout \& DiPrete 2006). Thus, the evaluation of the representativeness of SES in twins is important when generalizing the results from twin studies to the general population. One aspect of that validity assessment is to examine educational differences between MZ and DZ twins. There are at least three possible origins of differences between these two types of twins in terms of individual and parental education. First, because MZ twins tend to be shorter and weigh less at birth than DZ twins (Hur et al., 2005) and these birth-related factors may be associated with slower cognitive development (Broekman et al., 2009), it is possible that differences in IQ can be found between MZ and DZ twins that could lead to differences in academic performance in later life. This is supported by findings that twins have, in general, slightly lower IQs than singletons (Voracek \& Haubner, 2008), and this difference is even more pronounced in triplets, suggesting that there is a dose-response relationship between the birth-related anthropometrics of multiple pregnancies and later IQ (Silventoinen et al., 2013). However, this effect can at least partially be explained by birth order, as found in a Dutch study (de Zeeuw et al., 2012). There is also evidence that the multiple-birth effect on IQ has diminished over time (Silventoinen et al., 2013; Voracek \& Haubner, 2008), and it may not exist in the most recent birth cohorts (Calvin et al., 2009; Webbink et al., 2008). Previous studies on the zygosity differences in IQ from childhood through early adulthood have shown mixed results, with higher, similar, and lower IQ in MZ twins as compared with $\mathrm{DZ}$ twins without a clear age pattern (Haworth et al., 2009; Keller et al., 2013; Modig et al., 2011; Silventoinen et al., 2006). Furthermore, the IQ difference between $\mathrm{MZ}$ and $\mathrm{DZ}$ twins was small (i.e., less than three IQ points) in the reviewed studies, and thus is not likely to strongly affect academic performance.

Second, DZ twin births have become more common during the last decades in many countries because of the increasing use of in vitro fertilization and other infertility treatments (Imaizumi, 2003). A U.S. study found that mothers who have used fertility treatments - in vitro fertilization in particular - tend to be older, better educated, and are less likely to be smokers than those mothers who have not used these treatments (Tong et al., 2016). Higher maternal age and lower smoking rate, but not higher maternal education, were also found in a Dutch study of mothers who used in vitro fertilization (van Beijsterveldt et al., 2011), which may indicate differences in the access to in vitro fertilization procedures between countries. It is thus possible, especially in societies where fertility treatments are not publicly funded, that the socio-economic background of parents of DZ twins has improved relative to the parents of MZ 
twins since the 1980s when in vitro fertilization first became publicly available (Steptoe \& Edwards, 1976).

Third, it is possible that different social dynamics between MZ and DZ co-twins may lead to different educational outcomes. A Finnish study of adolescent twins found that MZ twins reported more dependency on their cotwin, and they spend more time together than DZ twins (Penninkilampi-Kerola et al., 2005). In that study, co-twin dependence was found to be associated with less ambitious academic careers after primary education, but otherwise it is poorly known whether this would affect educational differences between MZ and DZ twins.

The previous literature reviewed above suggests that both individual and parental education may differ between $\mathrm{MZ}$ and DZ twins and that these differences may have changed over time. We explored these potential differences in the present study by comparing $\mathrm{MZ}$ and $\mathrm{DZ}$ twins in a very large pooled twin database that contained information on individual, maternal, and paternal education from twin birth cohorts from the late 19th century through to the early 21 st century.

\section{Data and Methods}

The data were derived from the CODATwins (Collaborative project of Development of Anthropometrical Measures in Twins) database described in detail previously (Silventoinen et al., 2015). The project aimed to combine height and weight data from all twin projects in the world. In addition to the anthropometric measures, the collaborators were asked to provide data on individual education for adults and parental education for children. Together, we had information on individual education from 218,482 twin individuals from 27 twin cohorts representing 15 countries. Since we were interested how the zygosity differences changed over birth cohorts, we removed those without information on birth year (104 individuals), those born before 1890 (7 individuals), and those born after 2000 (9 individuals). Thus, in the analyses, we had 218,362 twin individuals with information on education (53\% females; 39\% MZ twins) including 95,208 twin pairs with information on education from both co-twins. Information on maternal education was available in 147,315 and paternal education in 143,056 twin individuals after excluding those without information on birth year (91 individuals for maternal and 89 individuals for paternal education) that came from 28 twin cohorts representing 15 countries ( $52 \%$ females; $38 \% \mathrm{MZ}$ twins). These twins come from 78,748 twin families for maternal and 76,024 twin families for paternal education.

Education classifications were transformed into education years using the average length of educational level in each country. The classifications for individual education for each cohort are presented in Supplementary Table S1 and for maternal and paternal education in Supplementary Table S2. Those who reported individual (2 cases), maternal (10 cases), or paternal (7 cases) education more than 22 years were coded to have 22 years of education (i.e., equivalent of $\mathrm{PhD}$ education).

The data were analyzed using linear regression models with individual or parental education as the dependent variable and zygosity and twin cohort as the independent variables. We stratified the analyses by 10 -year birth cohorts from 1890-1899 to 1990-1999 when analyzing individual education and to 2000 or later when analyzing maternal and paternal education. We first tested the main effect of zygosity on individual and parental education. In the analyses pooling all birth cohorts together, the results were additionally adjusted for 10 -year birth cohort by including it as a classified independent variable in the regression model to also take into account possible non-linear effects of birth cohort on individual or parental education. After that we tested whether the association between $z y-$ gosity and individual education is similar in males and females and whether the associations between zygosity and individual, maternal and paternal education have changed over the birth cohorts by fitting interaction terms between zygosity and sex as well as zygosity and birth cohort into the regression model. Thus, in total, we tested five interaction effects. When individual education was analyzed, we used twin individuals after taking into account the effect of sampling twin pairs rather than unrelated individuals on standard errors by using the cluster option of Stata/SE statistical software, version 13.1 for Windows (StataCorp, College Station, TX, USA). We also replicated the analyses for 172,970 twin individuals with information on education at 30 years of age or older to confirm that the results are similar if studying completed education. Furthermore, we analyzed this between same-sex and opposite-sex DZ twins using 201,949 twin individuals for whom we knew the sex of the co-twin. When we analyzed maternal and paternal education, only one twin from each family was selected since both co-twins have the same parental education.

As we had fewer families with information on paternal education than maternal education, we studied the representativeness of paternal education. We found that the maternal education was 0.56 (95\% CI $[0.47,0.66])$ years higher in families with information also available on paternal education as compared to families without information on paternal education, when adjusting the results for twin cohorts and 10-year birth cohorts. This suggests that in families of lower socio-economic position, it may be more likely that we did not have information on paternal education.

\section{Results}

Figure 1 presents the mean individual, maternal, and paternal education by birth cohort. The educational years increased over the birth cohorts and were higher for individual than for parental education, indicating the general educational transition in the world. An exception was the 


\section{$\mathrm{MZ}$ twins}

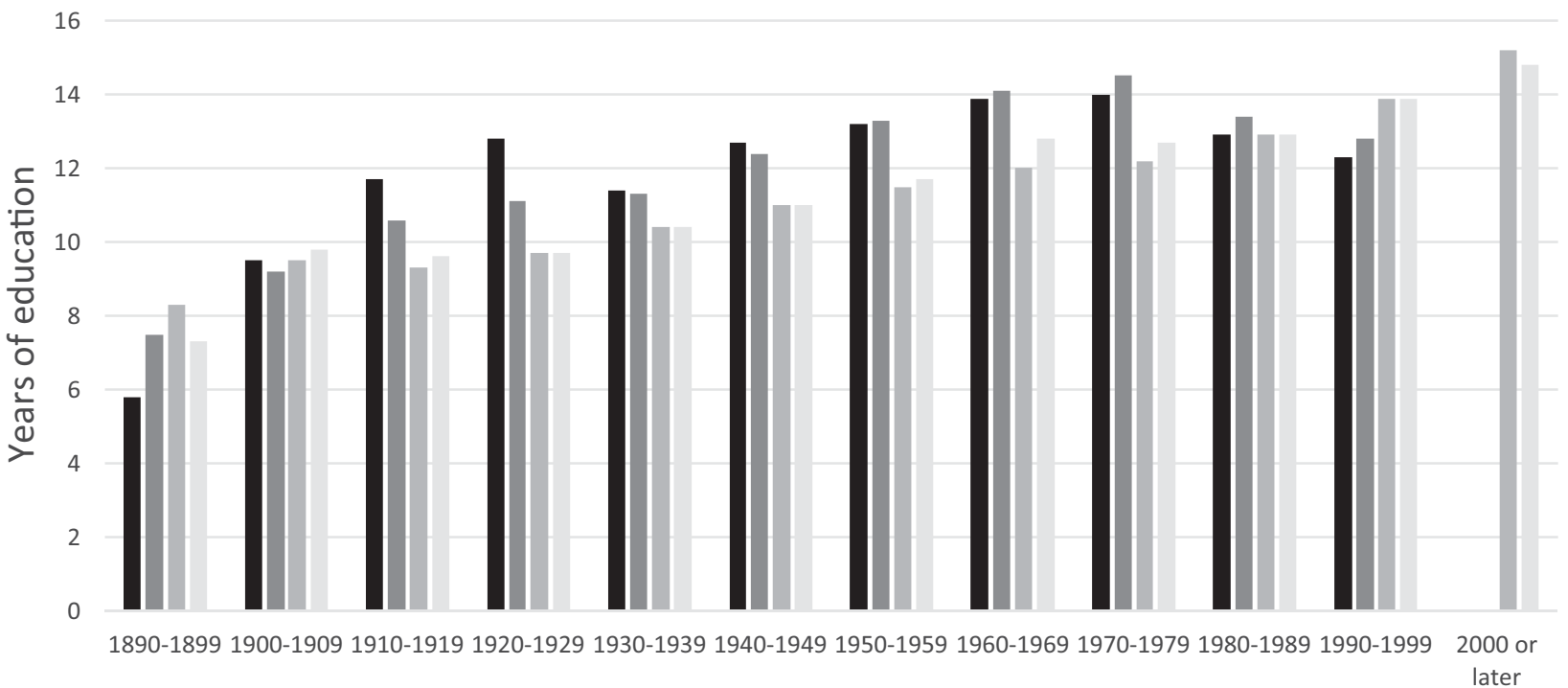

\section{DZ twins}

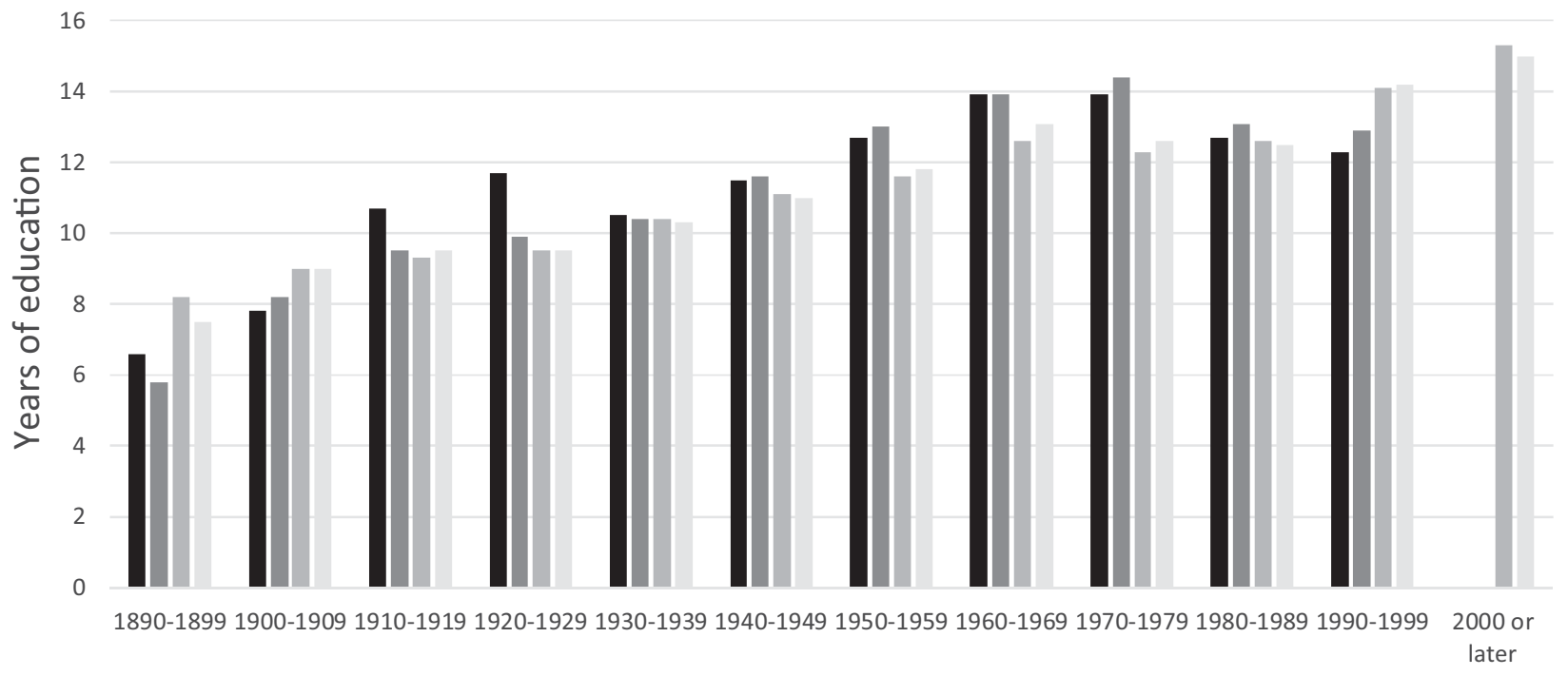

\section{Birth cohort}

Individual education in men $\square$ Individual education in women $\square$ Maternal education $\square$ Paternal education

\section{FIGURE 1}

Mean individual, maternal, and paternal education years by birth cohort.

cohort born 1990-1999, because in this cohort twins were generally younger and had not yet finalized their education.

We started the analyses by studying the zygosity difference in individual education. Among both men and women, MZ twins had slightly higher education levels than DZ twins (Table 1). This difference was seen in all birth cohorts except 1890-1899 in men and 1910-1909 and 19901999 in women, but according to linear regression, in some birth cohorts the zygosity difference was not statistically significant because of small sample size. When data pooled according to birth year were analyzed, a statistically significant interaction effect between sex and zygosity was found $(p<.0001)$ : in men, MZ twins had 0.26 (95\% CI $[0.21,0.31])$ years more education, whereas for women this difference was 0.17 (95\% CI [0.12, 0.21]) education years when the results were also additionally adjusted for birth cohort. However, there was also an interaction effect between zygosity and birth cohort $(p<.0001$ in both men and women): the education difference between twin types decreased, on average, by 0.09 years $(95 \%$ CI $[0.06,0.11])$ in 
TABLE 1

Number of Twin Individuals and Means, Standard Deviations (SD), and the Regression Coefficients ( $\beta$ ) With $95 \%$ Confidence Intervals (Cl) of Individual Education By Sex, Zygosity and Birth Cohort

\begin{tabular}{|c|c|c|c|c|c|c|c|c|}
\hline \multirow[b]{2}{*}{ Birth cohort } & \multicolumn{3}{|c|}{ MZ twins } & \multicolumn{3}{|c|}{ DZ twins } & \multicolumn{2}{|c|}{ Regression coefficient $^{1}$} \\
\hline & $N$ & Mean & $\mathrm{SD}$ & $N$ & mean & $D$ & $B$ & $95 \% \mathrm{Cl}$ \\
\hline \multicolumn{9}{|l|}{ Men } \\
\hline 1890-1899 & 27 & 5.8 & 3.94 & 41 & 6.6 & 3.81 & 0.32 & $-2.24,2.89$ \\
\hline 1900-1909 & 216 & 9.5 & 4.88 & 353 & 7.8 & 4.41 & -0.52 & $-1.09,0.05$ \\
\hline 1910-1919 & 1,585 & 11.7 & 4.16 & 2,286 & 10.7 & 4.28 & -0.41 & $-0.67,-0.15$ \\
\hline 1920-1929 & 6,294 & 12.8 & 3.79 & 8,988 & 11.7 & 4.15 & -0.25 & $-0.39,-0.12$ \\
\hline 1930-1939 & 3,139 & 11.4 & 4.35 & 7,417 & 10.5 & 4.46 & -0.12 & $-0.30,0.07$ \\
\hline 1940-1949 & 6,087 & 12.7 & 4.21 & 14,297 & 11.5 & 4.40 & -0.46 & $-0.59,-0.33$ \\
\hline 1950-1959 & 7,496 & 13.2 & 3.64 & 14,077 & 12.7 & 3.76 & -0.26 & $-0.37,-0.16$ \\
\hline 1960-1969 & 3,567 & 13.9 & 2.92 & 5,077 & 13.9 & 2.87 & -0.01 & $-0.15,0.12$ \\
\hline 1970-1979 & 4,900 & 14.0 & 2.71 & 5,683 & 13.9 & 2.58 & -0.18 & $-0.30,-0.06$ \\
\hline 1980-1989 & 3,948 & 12.9 & 2.54 & 5,117 & 12.7 & 2.47 & -0.13 & $-0.25,-0.02$ \\
\hline 1990-1999 & 593 & 12.3 & 1.96 & 665 & 12.3 & 2.21 & -0.15 & $-0.43,0.12$ \\
\hline \multicolumn{9}{|l|}{ Women } \\
\hline 1890-1899 & 57 & 7.5 & 4.15 & 75 & 5.8 & 2.88 & -0.05 & $-1.39,1.30$ \\
\hline 1900-1909 & 403 & 9.2 & 4.62 & 622 & 8.2 & 4.36 & -0.17 & $-0.62,0.27$ \\
\hline 1910-1919 & 1,528 & 10.6 & 4.03 & 2,378 & 9.5 & 4.09 & 0.01 & $-0.23,0.24$ \\
\hline 1920-1929 & 3,159 & 11.1 & 3.96 & 5,428 & 9.9 & 4.02 & -0.21 & $-0.38,-0.04$ \\
\hline 1930-1939 & 3,988 & 11.3 & 3.95 & 7,640 & 10.4 & 4.16 & -0.24 & $-0.40,-0.07$ \\
\hline 1940-1949 & 7,669 & 12.4 & 3.84 & 15,727 & 11.6 & 4.12 & -0.20 & $-0.31,-0.09$ \\
\hline 1950-1959 & 10,294 & 13.3 & 3.45 & 15,476 & 13.0 & 3.62 & -0.14 & $-0.23,-0.05$ \\
\hline 1960-1969 & 6,615 & 14.1 & 2.87 & 6,948 & 13.9 & 2.85 & -0.13 & $-0.24,-0.03$ \\
\hline 1970-1979 & 7,124 & 14.5 & 2.88 & 6,875 & 14.4 & 2.71 & -0.13 & $-0.24,-0.03$ \\
\hline 1980-1989 & 6,485 & 13.4 & 2.52 & 6,271 & 13.1 & 2.39 & -0.14 & $-0.24,-0.05$ \\
\hline 1990-1999 & 988 & 12.8 & 2.22 & 759 & 12.9 & 2.02 & 0.23 & $0.00,0.47$ \\
\hline
\end{tabular}

Note: ${ }^{1}$ Adjusted for twin cohort; $M Z$ twins used as the reference category.

men and by 0.10 years $(95 \%$ CI $[0.08,0.13])$ in women per 10-year birth cohort between 1890-1899 and 1990-1999. The comparisons between opposite-sex and same-sex DZ twins revealed no systematic differences, and in most of the birth cohorts the difference was non-significant (Supplementary Table S3). The analyses were repeated for participants 30 years of age or older using the pooled data to determine whether unfinished education affected the results. However, we found only slight changes $(0.29,95 \%$ CI $[0.24,0.35]$ education years difference in males and 0.19 , $95 \%$ CI $[0.14,0.23]$ education years difference in females when comparing $\mathrm{MZ}$ and $\mathrm{DZ}$ twins) as compared to the results using all twins. Furthermore, birth cohort-specific results were very similar except in the two latest birth cohorts for which there were not enough participants aged 30 or older to conduct the analyses (results not shown, but are available from the corresponding author).

We then conducted similar analyses for parental education (Table 2). When data from all birth cohorts were pooled together and the results were additionally adjusted for birth cohorts, no zygosity effect was seen for either maternal $(0.01,95 \%$ CI $[-0.03,0.06]$ years more education in $\mathrm{MZ}$ twins) or paternal education $(0.01,95 \% \mathrm{CI}[-0.04$, 0.05] years more education in MZ twins). We found some evidence of an interaction effect between zygosity and birth cohort both for maternal $(p=.001)$ and paternal education $(p<.0001)$ : the interaction term suggested that the zygosity difference in maternal education had changed by $0.03(95 \%$ CI $[0.01,0.04])$ years and paternal education by $0.05(95 \%$
CI $[0.03,0.07])$ years per 10 -year birth cohort. In the earliest birth cohorts, there was some evidence of higher maternal and paternal education in MZ twins, and the difference was statistically significant in the cohort born 1920-1929 $(0.31,95 \%$ CI $[0.13,0.48]$ years for maternal and $0.31,95 \%$ CI $[0.10,0.52]$ years for paternal education). However, this was no longer evident in the cohorts born after the 1950s. Instead, the fathers of DZ twins had higher education levels in the most recent cohorts born in 1990-1999 (0.16 95\% CI $[0.08,0.25]$ years $)$ and 2000 or later $(0.1195 \%$ CI [0.00, 0.22 ] years), but for maternal education we did not find a statistically significant difference.

\section{Discussion}

In this very large pooled twin study, we found that the education level of MZ twins was slightly higher than that of DZ twins. The difference was more pronounced in men and in the earliest birth cohorts, but even in these groups, the difference was quite small (less than 0.5 education years). We found some evidence of higher maternal and paternal education in MZ twins in the cohorts born in the 1950s or earlier, but paternal education was higher in DZ twins in the latest birth cohorts (1990-1999 and 2000 or later). The higher paternal education in these birth cohorts may be associated with the increased use of fertility treatments - in vitro fertilization in particular. U.S. mothers using in vitro fertilization tend to be older and better educated than other mothers (Tong et al., 2016), and 
TABLE 2

Number of Twin Families and Means, Standard Deviations, and Regression Coefficients ( $\beta$ ) With $95 \%$ Confidence Intervals $(\mathrm{Cl})$ of Maternal and Paternal Education By Zygosity and Birth Cohort

\begin{tabular}{|c|c|c|c|c|c|c|c|c|}
\hline \multirow[b]{2}{*}{ Birth cohort } & \multicolumn{3}{|c|}{ MZ twins } & \multicolumn{3}{|c|}{ DZ twins } & \multicolumn{2}{|c|}{ Regression coefficient $^{1}$} \\
\hline & $N$ & Mean & $S D$ & $N$ & mean & $S D$ & $\beta$ & $95 \% \mathrm{Cl}$ \\
\hline \multicolumn{9}{|c|}{ Maternal education } \\
\hline 1890-1899 & 8 & 8.3 & 2.49 & 5 & 8.2 & 3.90 & -0.05 & $-3.91,3.81$ \\
\hline 1900-1909 & 75 & 9.5 & 2.94 & 96 & 9.0 & 3.03 & -0.44 & $-1.36,0.47$ \\
\hline 1910-1919 & 713 & 9.3 & 3.07 & 826 & 9.3 & 2.91 & -0.03 & $-0.33,0.26$ \\
\hline 1920-1929 & 2,095 & 9.7 & 3.05 & 2,459 & 9.5 & 3.00 & -0.31 & $-0.48,-0.13$ \\
\hline 1930-1939 & 1,267 & 10.4 & 2.69 & 1,864 & 10.4 & 2.75 & -0.08 & $-0.27,0.11$ \\
\hline 1940-1949 & 2,794 & 11.0 & 2.62 & 4,763 & 11.1 & 2.72 & -0.11 & $-0.22,0.01$ \\
\hline 1950-1959 & 4,427 & 11.5 & 2.82 & 6,375 & 11.6 & 2.66 & -0.12 & $-0.22,-0.03$ \\
\hline 1960-1969 & 1,956 & 12.0 & 3.35 & 2,478 & 12.6 & 3.00 & 0.10 & $-0.06,0.27$ \\
\hline 1970-1979 & 2,451 & 12.2 & 3.37 & 2,997 & 12.3 & 3.17 & 0.10 & $-0.06,0.26$ \\
\hline 1980-1989 & 3,164 & 12.9 & 3.26 & 4,319 & 12.6 & 3.50 & 0.06 & $-0.09,0.21$ \\
\hline 1990-1999 & 7,009 & 13.9 & 2.90 & 12,909 & 14.1 & 2.92 & 0.06 & $-0.01,0.14$ \\
\hline 2000 or later & 4,280 & 15.2 & 3.04 & 9,418 & 15.3 & 3.18 & 0.06 & $-0.04,0.16$ \\
\hline \multicolumn{9}{|c|}{ Paternal education } \\
\hline 1890-1899 & 9 & 7.3 & 2.00 & 4 & 7.5 & 1.91 & 0.17 & $-2.44,2.78$ \\
\hline 1900-1909 & 67 & 9.8 & 3.69 & 89 & 9.0 & 3.37 & -0.82 & $-1.95,0.31$ \\
\hline 1910-1919 & 675 & 9.6 & 3.49 & 800 & 9.5 & 3.47 & -0.05 & $-0.40,0.31$ \\
\hline 1920-1929 & 2,018 & 9.7 & 3.65 & 2,378 & 9.5 & 3.42 & -0.31 & $-0.52,-0.10$ \\
\hline 1930-1939 & 1,219 & 10.4 & 3.08 & 1,781 & 10.3 & 3.06 & -0.08 & $-0.30,0.14$ \\
\hline 1940-1949 & 2,724 & 11.0 & 3.07 & 4,626 & 11.0 & 3.19 & -0.06 & $-0.21,0.08$ \\
\hline 1950-1959 & 4,290 & 11.7 & 3.34 & 6,224 & 11.8 & 3.24 & -0.18 & $-0.30,-0.06$ \\
\hline 1960-1969 & 1,869 & 12.8 & 3.66 & 2,365 & 13.1 & 3.36 & -0.05 & $-0.25,0.16$ \\
\hline 1970-1979 & 2,308 & 12.7 & 3.48 & 2,765 & 12.6 & 3.35 & 0.07 & $-0.11,0.24$ \\
\hline 1980-1989 & 2,980 & 12.9 & 3.60 & 4,090 & 12.5 & 3.88 & 0.08 & $-0.08,0.24$ \\
\hline 1990-1999 & 6,875 & 13.9 & 3.03 & 12,665 & 14.2 & 3.06 & 0.16 & $0.08,0.25$ \\
\hline 2000 or later & 4,114 & 14.8 & 3.34 & 9,089 & 15.0 & 3.34 & 0.11 & $0.00,0.22$ \\
\hline
\end{tabular}

Note: ${ }^{1}$ Adjusted for twin cohort; $M Z$ twins used as the reference category.

this, in turn, may also have affected paternal education because of educational homogamy, which is well known in many societies (Blossfeld, 2009). Also, the fertility treatment is expensive, and a husband's income determines the social position of the family in many societies, which may explain why the effect is particularly evident in paternal education.

The observation that MZ twins had slightly higher education than DZ twins is puzzling. We found some evidence of higher parental education in the earliest birth cohorts, but this effect disappeared in the later birth cohorts and even reversed for paternal education, thus not supporting the idea that the difference in individual education would be caused by socio-economic background. It is also not very likely that physiological features related to twin pregnancies would be the explanation. MZ twins are somewhat lighter at birth (Hur et al., 2005) and slightly shorter in adolescence and adulthood than DZ twins (Jelenkovic et al., 2015). Low birth weight has been found to be associated with slower cognitive development (Broekman et al., 2009) and short stature in adulthood with lower IQ (Silventoinen et al., 2006) and less education (Magnusson et al., 2006). Thus, the zygosity differences in birth size and later height would predict an effect in the opposite direction of what was found.

One explanation for the slightly higher education in $\mathrm{MZ}$ as compared with DZ twins could be different social dynamics within $\mathrm{MZ}$ and $\mathrm{DZ}$ co-twins. In a Finnish study,
MZ twins reported more dependence on the co-twin than did DZ twins, but this was related to selecting a vocational rather than an academic educational path after the compulsory primary education (Penninkilampi-Kerola et al., 2005). There is also some evidence that cooperation is more common in MZ than in DZ twin pairs (Segal, 2002; Segal \& Hershberger, 1999). More cooperation and a greater similarity in intelligence in $\mathrm{MZ}$ than $\mathrm{DZ}$ twins might help $\mathrm{MZ}$ twins continue schooling together. However, it is clear that more research is needed to find out whether this could explain the observed zygosity difference in education years.

Still another possible explanation of the differences in education between $\mathrm{MZ}$ and $\mathrm{DZ}$ twins could be differences in maternal age also affecting birth order. It is well known that older maternal age not only increases DZ births because of the increasing use of in vitro fertilization but also natural DZ twinning rates (Derom et al., 2011). Thus, it is also likely that $\mathrm{DZ}$ twins more often have later parity than MZ twins. Older maternal age has been found to be associated with slightly lower IQ when adjusted for birth cohort effect (Myrskylä et al., 2013), and the number of older siblings also has a negative effect on education (Black et al., 2005; Brooth \& Kee, 2009). Because fertility has decreased during the 20th century (Lesthaeghe, 2010), this effect may have become weaker as the average family size has decreased, which parallels our result on the decreasing difference in education between MZ and DZ twins over the birth cohorts. 
It is also possible that selective participation may have affected our results. Higher than expected proportions of MZ twins have been found in many twin cohorts suggesting that participation rates have been higher in MZ than DZ twins (Silventoinen et al., 2015), and those in higher socioeconomic positions tend to more actively take part in surveys in general (Laaksonen et al., 2008). This may have led to the situation that $\mathrm{DZ}$ twins in the surveys are more socially selected than MZ twins. Selective participation due to differential mortality or disease occurrence could also explain these findings. Monochorionic twins, who are always MZ, have higher perinatal mortality than dichorionic twins (Oldenburg et al., 2012). Thus, we can speculate that the MZ twins who have both survived are more robust and may obtain higher education levels. This may also explain the higher parental education in MZ twins born before World War II. Self-selection in the participating twin surveys has probably also affected our results in another way. It is unlikely that twins suffering from serious birth-related effects, such as cerebral palsy, took part in the surveys. These defects are much more common in monochorionic than in dichorionic twins (Pharoah \& Dundar, 2009), and the likely lower participation rates of these twins are thus more likely to create bias for MZ than DZ twins. Our results should thus be generalized primarily to the healthy twin populations without any serious birth-related complications affecting school performance.

Our data do not include information on singletons, and thus we cannot study whether twins differ from singletons according to their educational achievement. Previous studies on this issue have produced somewhat conflicting results. A Taiwanese study found that both test scores and the probability to attend college were lower in twins than singletons (Tsou et al., 2008). On the other hand, studies from Denmark (Christensen et al., 2006) and the Netherlands (de Zeeuw et al., 2012) did not find differences in educational achievement between twins and singletons, and a Swedish study found that twins had slightly better educational achievement than singletons (Hjern et al., 2012). It is thus likely that twins do not have poorer academic achievement in Western countries, but it is too early to argue whether this also applies to East Asia. Furthermore, in all of these previous studies, the participants were born in the 1970s or later. Since there is clear evidence of the trend of lower IQs in twins compared to singletons in the earlier birth cohorts diminishing in the more recent birth cohorts (Silventoinen et al., 2013; Voracek \& Haubner, 2008), it is possible that twins have also been behind singletons in school performance in the earlier birth cohorts.

Our data have both strengths and weaknesses. Our main strength is the very large sample size, allowing us to convincingly demonstrate even the very small difference in education levels between MZ and DZ twins. Such small differences would be difficult to find in any of the existing twin cohorts alone. We also had information on the ma- ternal and paternal education of twins. It is also an advantage that we have twin birth cohorts over a period of more than 100 years, allowing us to study temporal changes of the zygosity differences. One limitation is that we do not have information on the academic performance of the twins at school; so, we do not know whether the difference in education is due to better school performance or rather continuing education with lower grades. Also, we do not have information on singletons and thus cannot say how the education of MZ and DZ twins compares to the general population. Furthermore, we do not have any information on maternal age and the number of older siblings, which may affect educational differences between MZ and DZ twins. We also found some evidence that paternal education may be selective since maternal education was higher in families where we also had information on paternal education than in families where this information was missing. Finally, pooling data from twin cohorts representing different countries and birth cohorts creates challenges when harmonizing education classifications. This is partly related to different ways to ask about education in the surveys - some cohorts have used only a few education levels, whereas others have used the exact years of education - but also reflects large differences in educational systems between countries and over time. Thus, we have focused only on education adjusted by twin cohort and birth cohort and consequently relative rather than absolute education.

In conclusion, $\mathrm{MZ}$ twins have slightly but systematically higher education than $\mathrm{DZ}$ twins, and this difference is more pronounced in men and in earlier birth cohorts. The difference is, however, so small that it is not likely to affect the comparability of $\mathrm{MZ}$ and $\mathrm{DZ}$ twins when studying the heritability of education or applying the twin design to other research questions. If this difference is regarded as a problem, then special care should be paid to make $\mathrm{MZ}$ and $\mathrm{DZ}$ twins comparable for parity, family size, maternal age, and other factors that may differ between MZ and DZ twins and in turn affect education. For parental education, we found only minor and unsystematic differences between MZ and DZ twins. Thus, our results suggest that the social background of MZ and DZ twins is largely comparable.

\section{Acknowledgments}

This study was conducted within the CODATwins project (Academy of Finland \#266592). The Australian Twin Registry is supported by a Centre of Research Excellence (grant ID 1079102) from the National Health and Medical Research Council administered by the University of Melbourne. The Boston University Twin Project is funded by grants (\#R01 HD068435 \#R01 MH062375) from the National Institutes of Health to K. Saudino. California Twin Program was supported by The California Tobacco-Related Disease Research Program (7RT-0134H, 8RT-0107H, 6RT-0354H) and the National Institutes of 
Health (1R01ESO15150-01). The Carolina African American Twin Study of Aging (CAATSA) was funded by a grant from the National Institute on Aging (grant 1RO1AG13662-01A2) to K. E. Whitfield. The CATSS-Study is supported by the Swedish Research Council through the Swedish Initiative for Research on Microdata in the Social And Medical Sciences (SIMSAM) framework grant no 340-2013-5867, grants provided by the Stockholm County Council (ALF-projects), the Swedish Heart-Lung Foundation and the Swedish Asthma and Allergy Association's Research Foundation. Colorado Twin Registry is funded by NIDA funded center grant DA011015 and Longitudinal Twin Study HD10333; Author Huibregtse is supported by 5T32DA017637-11. Since its origin, the East Flanders Prospective Survey has been partly supported by grants from the Fund of Scientific Research, Flanders and Twins, a non-profit Association for Scientific Research in Multiple Births (Belgium). Data collection and analyses in Finnish twin cohorts have been supported by ENGAGE - European Network for Genetic and Genomic Epidemiology, FP7-HEALTH-F4-2007, grant agreement number 201413, National Institute of Alcohol Abuse and Alcoholism (grants AA-12502, AA-00145, and AA-09203 to R J Rose, the Academy of Finland Center of Excellence in Complex Disease Genetics (grant numbers: 213506, 129680), and the Academy of Finland (grants 100499, $205585,118555,141054,265240,263278$, and 264146 to J Kaprio). Gemini was supported by a grant from Cancer Research UK (C1418/A7974). Anthropometric measurements of the Hungarian twins were supported by Medexpert Ltd., Budapest, Hungary. Korean Twin-Family Register was supported by the Global Research Network Program of the National Research Foundation (NRF 2011-220-E00006). Longitudinal Israeli Study of Twins was funded by the Starting Grant no. 240994 from the European Research Council (ERC) to Ariel Knafo. The Michigan State University Twin Registry has been supported by Michigan State University, as well as grants R01-MH081813, R01-MH0820-54, R01-MH092377-02, R21-MH070542-01, R03-MH6385101 from the National Institute of Mental Health (NIMH), R01-HD066040 from the Eunice Kennedy Shriver National Institute for Child Health and Human Development (NICHD), and 11-SPG-2518 from the MSU Foundation. The content of this manuscript is solely the responsibility of the authors and does not necessarily represent the official views of the NIMH, the NICHD, or the National Institutes of Health. The Murcia Twin Registry is supported by Fundación Séneca, Regional Agency for Science and Technology, Murcia, Spain (08633/PHCS/08, 15302/PHCS/10 \& 19479/PI/14) and Ministry of Science and Innovation, Spain (PSI2009-11560 \& PSI2014-56680R). The NAS-NRC Twin Registry acknowledges financial support from the National Institutes of Health grant number R21 AG039572. Netherlands Twin Register acknowledges the Netherlands Organization for Scientific Research
(NWO) and MagW/ZonMW grants 904-61-090, 985-10002, 912-10-020, 904-61-193,480-04-004, 463-06-001, 45104-034, 400-05-717, Addiction-31160008, Middelgroot911-09-032, Spinozapremie 56-464-14192; VU University's Institute for Health and Care Research (EMGO+); the European Research Council (ERC - 230374), the Avera Institute, Sioux Falls, South Dakota (USA). Madeira data comes from the following project: Genetic and environmental influences on physical activity, fitness, and health: the Madeira family study. Project reference: POCI/DES/56834/2004. Founded by the Portuguese agency for research (The Foundation for Science and Technology [FCT]). South Korea Twin Registry is supported by National Research Foundation of Korea (NRF-371-20111 B00047). The Texas Twin Project is currently funded by grants AA023322 and HD081437 from the National Institutes of Health. S. Y. Öncel and F. Aliev are supported by Kırıkkale University Research Grant: KKU, 2009/43 and TUBITAK grant 114C117. Washington State Twin Registry (formerly the University of Washington Twin Registry) was supported in part by grant NIH RC2 HL103416 (D. Buchwald, PI). Vietnam Era Twin Study of Aging was supported by National Institute of Health grants NIA R01 AG018384, R01 AG018386, R01 AG022381, and R01 AG022982, and, in part, with resources of the VA San Diego Center of Excellence for Stress and Mental Health. The Cooperative Studies Program of the Office of Research \& Development of the United States Department of Veterans Affairs has provided financial support for the development and maintenance of the Vietnam Era Twin (VET) Registry. The content of this manuscript is solely the responsibility of the authors and does not necessarily represent the official views of the NIA/NIH, or the VA. The West Japan Twins and Higher Order Multiple Births Registry was supported by Grant-in-Aid for Scientific Research (B) (grant number 15H05105) from the Japan Society for the Promotion of Science. The University of Southern California Twin Study is funded by a grant from the National Institute of Mental Health (R01 MH58354). Osaka University Aged Twin Registry is supported by grants from JSPS KAKENHI JP (23593419, 24792601, 26671010, 24590695, 26293128, 16K15385, 16K15978, 16K15989, 16H03261).

\section{Conflict of Interest}

None.

\section{Supplementary Material}

To view supplementary material for this article, please visit https://doi.org/10.1017/thg.2017.49

\section{References}

Black, S. E., Devereux, P. J., \& Salvanes, K. G. (2005). The more the merrier? The effect of family size and birth order on 
children's education. Quarterly Journal of Economics, 120, 669-700.

Blossfeld, H. (2009). Educational assortative marriage in comparative perspective. Annual Review of Sociology, 35, 513530.

Broekman, B. F., Chan, Y. H., Chong, Y. S., Quek, S. C., Fung, D., Low, Y.L., ... Saw, S. M. (2009). The influence of birth size on intelligence in healthy children. Pediatrics, 123, e1011-e1016.

Brooth, A. L., \& Kee, H. J. (2009). Birth order matters: The effect of family size and birth order on educational attainment. Journal of Population Economics, 22, 367-397.

Calvin, C., Fernandes, C., Smith, P., Visscher, P. M., \& Deary, I. J. (2009). Is there still a cognitive cost of being a twin in the UK? Intelligence, 37, 243-248.

Christensen, K., Petersen, I., Skytthe, A., Herskind, A. M., McGue, M., \& Bingley, P. (2006). Comparison of academic performance of twins and singletons in adolescence: Follow-up study. BMJ, 333, no. 1095.

de Zeeuw, E. L., van Beijsterveldt, C. E. M. M., de Geus, E. J., \& Boomsma, D. I. (2012). Twin specific risk factors in primary school achievements. Twin Research and Human Genetics, 15, 107-115.

Derom, C., Gielen, M., Peeters, H., Frijns, J. P., \& Zeegers, M. P. (2011). Time trends in the natural dizygotic twinning rate. Human Reproduction, 26, 2247-2252.

Haworth, C. M., Wright, M. J., Martin, N. W., Martin, N. G., Boomsma, D. I., Bartels, M., ... Plomin, R. (2009). A twin study of the genetics of high cognitive ability selected from 11,000 twin pairs in six studies from four countries. Behavior Genetics, 39, 359-370.

Hjern, A., Ekeus, C., Rasmussen, F., \& Lindblad, F. (2012). Educational achievement and vocational career in twins - a Swedish national cohort study. Acta Paediatrica, 101, 591596.

Hout, M., \& DiPrete, T. A. (2006). What we have learned: RC28's contributions to knowledge about social stratification. Research in Social Stratification and Mobility, 24, 1-20.

Hur, Y. M., Luciano, M., Martin, N. G., Boomsma, D. I., Iacono, W. G., McGue, M., ... Han, J. Y. (2005). A comparison of twin birthweight data from Australia, the Netherlands, the United States, Japan, and South Korea: Are genetic and environmental variations in birthweight similar in Caucasians and East Asians? Twin Research and Human Genetics, 8, 638-648.

Imaizumi, Y. (2003). A comparative study of zygotic twinning and triplet rates in eight countries, 1972-1999. Journal of Biosocial Science, 35, 287-302.

Jelenkovic, A., Yokoyama, Y., Sund, R., Honda, C., Bogl, L. H., Aaltonen, S., ... Silventoinen, K. (2015). Zygosity differences in height and body mass index of twins from infancy to old age: A study of the CODATwins project. Twin Research and Human Genetics, 18, 557-570.

Keller, M. C., Garver-Apgar, C. E., Wright, M. J., Martin, N. G., Corley, R. P., Stallings, M. C., ... Zietsch, B. P. (2013). The genetic correlation between height and IQ: Shared genes or assortative mating? PLoS Genetics, 9, no. e1003451.
Laaksonen, M., Aittomäki, A., Lallukka, T., Rahkonen, O., Saastamoinen, P., Silventoinen, K., \& Lahelma, E. (2008). Register-based study among employees showed small nonparticipation bias in health surveys and check-ups. Journal of Clinical Epidemiology, 61, 900-906.

Lesthaeghe, R. (2010). The unfolding story of the second demographic transition. Population and Development Review, 36, 211-251.

Mackenbach, J. P., Stirbu, I., Roskam, A. J., Schaap, M. M., Menvielle, G., Leinsalu, M., Kunst, A. E., \& European Union Working Group on Socioeconomic Inequalities in Health. (2008). Socioeconomic inequalities in health in 22 European countries. New England Journal of Medicine, 358, 2468-2481.

Magnusson, P. K., Rasmussen, F., \& Gyllensten, U. B. (2006). Height at age 18 years is a strong predictor of attained education later in life: Cohort study of over 950,000 Swedish men. International Journal of Epidemiology, 35, 658-663.

Modig, K., Silventoinen, K., Tynelius, P., Kaprio, J., \& Rasmussen, F. (2011). Genetics of the association between intelligence and nicotine dependence: A study of male Swedish twins. Addiction, 106, 995-1002.

Myrskylä, M., Silventoinen, K., Tynelius, P., \& Rasmussen, F. (2013). Is later better or worse? Association of advanced parental age with offspring cognitive ability among half a million young Swedish men. American Journal of Epidemiology, 177, 649-655.

Oldenburg, A., Rode, L., Bødker, B., Ersbak, V., Holmskov, A., Jørgensen, F. S., ... Tabor, A. (2012). Influence of chorionicity on perinatal outcome in a large cohort of Danish twin pregnancies. Ultrasound in Obstetrics and Gynecology, 39, 69-74.

Penninkilampi-Kerola, V., Moilanen, I., \& Kaprio, J. (2005). Co-twin dependence social interactions and academic achievement - A population-based study of adolescent twins and their parents. Journal of Social and Personal Relationships, 22, 519-541.

Pharoah, P. O., \& Dundar, Y. (2009). Monozygotic twinning, cerebral palsy and congenital anomalies, Hum Reprod Update, 15, 639-648.

Posthuma, D., Beem, A. L., de Geus, E. J., van Baal, G. C., von Hjelmborg, J. B., Iachine, I., \& Boomsma, D. I. (2003). Theory and practice in quantitative genetics. Twin Research, 6 , 361-376.

Segal, N. L. (2002). Co-conspiration and double dealers: A twin film analysis. Personality and Individual Differences, 33, 621-631.

Segal, N. L., \& Hershberger, S. L. (1999). Cooperation and competition in adolecents twins: Findings from a prisoner's dilemma game. Evolution and Human Behavior, 20, 29-51.

Silventoinen, K., Jelenkovic, A., Sund, R., Honda, C., Aaltonen, S., Yokoyama, Y., ... Kaprio, J. (2015). The CODATwins Project: The cohort description of collaborative project of development of anthropometrical measures in twins to study macro-environmental variation in genetic and environmental effects on anthropometric traits. Twin Research and Human Genetics, 18, 348-360. 
Silventoinen, K., Magnusson, P. K., Tynelius, P., Kaprio, J., \& Rasmussen, F. (2008). Heritability of body size and muscle strength in young adulthood: A study of one million Swedish men. Genetic Epidemiology, 32, 341-349.

Silventoinen, K., Myrskylä, M., Tynelius, P., Yokoyama, Y., \& Rasmussen, F. (2013). Social modifications of the multiple birth effect on IQ and body size: A population-based study of young adult males. Paediatric and Perinatal Epidemiology, 27, 380-387.

Silventoinen, K., Posthuma, D., van Beijsterveldt, T., Bartels, M., \& Boomsma, D. I. (2006). Genetic contributions to the association between height and intelligence: Evidence from Dutch twin data from childhood to middle age. Genes, Brain, and Behavior, 5, 585-595.

Steptoe, P. C., \& Edwards, R. C. (1976). Reimplantation of a human embryo with subsequent tubal pregnancy. Lancet, 2(8085), 880-882.

Tong, V. T., Kissin, D. M., Bernson, D., Copeland, G., Boulet, S. L., Zhang, Y., ... England, L. J. (2016). Maternal smok- ing among women with and without use of assisted reproductive technologies. Journal of Womens Health, 25, 10661072.

Tsou, M. T., Tsou, M. W., Wu, M. P., \& Liu, J. T. (2008). Academic achievement of twins and singletons in early adulthood: Taiwanese cohort study. BMJ, 337, a438.

van Beijsterveldt, C. E. M., Bartels, M., \& Boomsma, D. I. (2011). Comparison of naturally conceived and IVFDZ twins in the Netherlands Twin Registry: A developmental study. Journal of Pregnancy, Article ID 517614.

Voracek, M., \& Haubner, T. (2008). Twin-singleton differences in intelligence: A meta-analysis, Psychological Reports, 102, 951-962.

Webbink, D., Posthuma, D., Boomsma, D.I., de Geus, E. J. C., \& Visscher, P. M. (2008). Do twins have lower cognitive ability than singletons? Intelligence, 36, 539-547. 